\title{
PRIME RINGS WITH ANNIHILATOR CONDITIONS ON POWER VALUES OF DERIVATIONS ON MULTILINEAR POLYNOMIALS
}

\author{
Vincenzo De Filippis
}

\begin{abstract}
Let $R$ be a prime algebra over a commutative ring $K, d$ a nonzero derivation of $R, f\left(x_{1}, . ., x_{n}\right)$ a multilinear polynomial over $K$ in $n$ non-commuting variables, $a \in R$ and $m \geq 1$ a fixed integer. Suppose that $f\left(x_{1}, . ., x_{n}\right)$ is not central on $R$. If $a\left(d\left(f\left(r_{1}, . ., r_{n}\right)\right)\right)^{m}=0$, for any $r_{1}, \ldots, r_{n} \in R$, then $a=0$.
\end{abstract}

Throughout this paper $R$ always denotes a prime ring with center $Z(R)$ and with extended centroid $C, Q$ its Martindale quotient ring. We will consider some related problems concerning annihilators of power values of derivations in prime rings.

In [2] $\mathrm{M}$. Bresar proved that if $R$ is a semiprime ring, $d$ a nonzero derivation of $R$ and $a \in R$ such that $a d(x)^{m}=0$, for all $x \in R$, where $m$ is a fixed integer, then $\operatorname{ad}(R)=0$ when $R$ is $(m-1)$ !-torsion free. In [9] T. K. Lee and J. S. Lin proved Bresar's result whitout the assumption of $(m-1)$ !-torsion free on $R$. They studied the Lie ideal case and, for the prime case, they showed that if $R$ is a prime ring with a derivation $d \neq 0, L$ a Lie ideal of $R, a \in R$ such that $a d(u)^{m}=0$, for all $u \in L$, where $m$ is fixed, then $\operatorname{ad}(L)=0$ unless the case when $\operatorname{char}(R)=2$ and $\operatorname{dim}_{C} R C=4$. In addition, if $[L, L] \neq 0$, then $\operatorname{ad}(R)=0$.

Recently in [3] C. M. Chang and T. K. Lee established a unified version of the previous results for prime rings. More precisely they proved the following theorem: let $R$ be a prime ring, $\varrho$ a nonzero right ideal of $R, d$ a nonzero derivation of $R$, $a \in R$ such that $a d([x, y])^{m} \in Z(R)\left(d([x, y])^{m} a \in Z(R)\right)$. If $[\varrho, \varrho] \varrho \neq 0$ and $\operatorname{dim}_{C} R C>4$, then either $a d(\varrho)=0(a=0$ resp. $)$ or $d$ is the inner derivation induced by some $q \in Q$ such that $q \varrho=0$.

Here we shall continue the investigation about the properties of a subset $S$ of $R$ related to its left annihilator $A n n_{R}(S)=\{x \in R: x S=(0)\}$. More precisely

Received June 10, 1999; revised Oct. 19, 1999.

Communicated by Pjek-Hwee Lee.

2000 Mathematics Subject Classification: 16N60, 16W25.

Key words and phrases: Prime rings, Differential identity, generalieed polynomial idenntity. 
we shall study the case when $S=\left\{d\left(f\left(x_{1}, . ., x_{n}\right)\right)^{m}: x_{1}, . ., x_{n} \in R\right\}$, where $f\left(x_{1}, . ., x_{n}\right)$ is a multilinear polynomial in $\mathrm{n}$ non-commuting variables and $m$ is a fixed integer. We shall prove:

Theorem 1. Let $R$ be a prime algebra over a commutative ring $K, d$ a nonzero derivation of $R, f\left(x_{1}, . ., x_{n}\right)$ a multilinear polynomial over $K$ in $n$ non-commuting variables, $a \in R, m \geq 1$ a fixed integer. Suppose that $f\left(x_{1}, . ., x_{n}\right)$ is not central on $R$. If $a\left(d\left(f\left(r_{1}, . ., r_{n}\right)\right)\right)^{m}=0$, for any $r_{1}, . ., r_{n} \in R$, then $a=0$.

We first dispose of the case that $R$ is not a domain. In fact, if $R$ is a domain, by supposing $a \neq 0$, we get $\left(d\left(f\left(r_{1}, . ., r_{n}\right)\right)\right)^{m}=0$, for any $r_{1}, . ., r_{n} \in R$. In this situation, by [13], $f\left(x_{1}, . ., x_{n}\right)$ must be central on $R$.

In all that follows let $T=Q{ }^{*} C C\{X\}$ be the free product over $C$ of the $C$-algebra $Q$ and the free $C$-algebra $C\{X\}$, with $X$ the countable set consisting of non-commuting indeterminates $x_{1}, x_{2}, \ldots, x_{n}, \ldots$ We refer the reader to [4] for the definitions and the related properties of these objects. Moreover we must remark that the main tool will be the theory of differential identities, initiated by Kharchenko in [6].

Remark 1. Recall that $d$ can be extended uniquely to a derivation on $Q$ [8] which will be also denoted by $d$. Since by [8] $R$ and $Q$ satisfy the same differential identities, we have that $a\left(d\left(f\left(x_{1}, . ., x_{n}\right)\right)\right)^{m}=0$ also in $Q$. Moreover $Q$ is prime, by the primeness of $R$, and replacing $R$ by $Q$ we may assume, without loss of generality, $C=Z(R)$ and $R$ is a $C$-algebra centrally closed.

From now on let $K$ be a commutative ring, $R$ a prime $K$-algebra, $f\left(x_{1}, . ., x_{n}\right)$ a multilinear polynomial over $K$ in $n$ non-commuting variables, $a \in R$ and $m \geq 1$.

Moreover $f\left(x_{1}, . ., x_{n}\right)$ is not central on $R$ and, for all $r_{1}, . ., r_{n} \in R, a\left(d\left(f\left(r_{1}, . .\right.\right.\right.$, $\left.\left.\left.r_{n}\right)\right)\right)^{m}=0$. We will use the following notation:

$$
f\left(x_{1}, . ., x_{n}\right)=x_{1} x_{2} . . x_{n}+\sum_{\sigma \in S_{n}} \alpha_{\sigma} x_{\sigma(1)} x_{\sigma(2)} \ldots x_{\sigma(n)} .
$$

We begin with the following:

Lemma 1. If $d$ is an outer derivation of $R$ then $a=0$.

Proof. Suppose on the contrary that $a \neq 0$. We denote by $f^{d}\left(x_{1}, . ., x_{n}\right)$ the polynomial obtained from $f\left(x_{1}, . ., x_{n}\right)$ by replacing each coefficient $\alpha_{\sigma}$ with $\delta\left(\alpha_{\sigma}\right.$. $1)$. Thus we write $d\left(f\left(x_{1}, . ., x_{n}\right)\right)=f^{d}\left(x_{1}, . ., x_{n}\right)+\sum_{i} f\left(x_{1}, . ., d\left(x_{i}\right), . ., x_{n}\right)$. Since $R$ satisfies the generalized differential identity

$$
a\left(d\left(f\left(x_{1}, . ., x_{n}\right)\right)\right)^{m}=
$$




$$
a\left(f^{d}\left(x_{1}, . ., x_{n}\right)+\sum_{i} f\left(x_{1}, . ., d\left(x_{i}\right), . ., x_{n}\right)\right)^{m}
$$

and $d$ is an outer derivation, by [6] $R$ satisfies the generalized polynomial identity

$$
a\left(f^{d}\left(x_{1}, . ., x_{n}\right)+\sum_{i} f\left(x_{1}, . ., y_{i}, . ., x_{n}\right)\right)^{m}
$$

and in particular $R$ satisfies $a\left(f\left(y_{1}, x_{2}, . ., x_{n}\right)\right)^{m}$. As a consequence of [5], since $R$ is prime and $f\left(x_{1}, . ., x_{n}\right)$ is not an identity for $R$, we get $a=0$, a contradiction.

In all that follows we will consider the only case when $d$ is an inner derivation in $R$. This means that there exists $q \in R$ such that $a\left[q, f\left(r_{1}, . ., r_{n}\right)\right]^{m}=0$, for any $r_{1}, . ., r_{n} \in R$.

Lemma 2. If $R$ does not satisfy any non-trivial generalized polynomial identity, then $a=0$.

Proof. Since $R$ does not satisfy any non-trivial generalized polynomial identity, we have that

$$
a\left[q, f\left(x_{1}, . ., x_{n}\right)\right]^{m}
$$

is the zero element in the free product $T=Q *_{C} C\left\{x_{1}, . ., x_{n}\right\}$, that is

$$
a\left[q, f\left(x_{1}, . ., x_{n}\right)\right]^{m-1}\left(q f\left(x_{1}, . ., x_{n}\right)-f\left(x_{1}, . ., x_{n}\right) q\right)=0 \in T .
$$

Since $q \notin C$, it follows that $a\left[q, f\left(x_{1}, . ., x_{n}\right)\right]^{m-1} f\left(x_{1}, . ., x_{n}\right) q=0 \in T$ and so $a\left[q, f\left(x_{1}, . ., x_{n}\right)\right]^{m-1}=0 \in T$. Continuing this process, we obtain that $a=0$.

Lemma 3. If $R$ is a dense ring of linear transformations over an infinite dimensional right vector space $V$ over a division ring $D$, then $a=0$.

Proof. Since $f\left(x_{1}, . ., x_{n}\right)$ is a multilinear polynomial and $a\left[q, f\left(r_{1}, . ., r_{n}\right)\right]^{m}=$ 0 , for all $r_{1}, . ., r_{n} \in R$, by [13, Lemma 2] we have $a[q, r]^{m}=0$, for all $r \in R$. Hence $a=0$ follows from [9, Theorem 1].

Now we are ready to prove the following:

Theorem 1. Let $R$ be a prime $K$-algebra, $d$ a nonzero derivation of $R$, $f\left(x_{1}, . ., x_{n}\right)$ a multilinear polynomial over $K$ in $n$ non-commuting variables, $a \in R$ and $m \geq 1$. Suppose that $f\left(x_{1}, . ., x_{n}\right)$ is not central on $R$. If $a\left(d\left(f\left(r_{1}, . ., r_{n}\right)\right)^{m}=\right.$ 0 , for any $r_{1}, . ., r_{n} \in R$, then $a=0$. 
Proof. By Lemma 1, we assume that $d$ is the inner derivation induced by $q \in R$, moreover by remark $1, C=Z(R)$ and $R$ is a $C$-algebra centrally closed, that is $R=R C$. If $R$ does not satisfy any non-trivial generalized polynomial identity then, by Lemma $2, a=0$. Thus we may suppose that $R$ satisfies a non-trivial generalized polynomial identity. By Martindale's theorem in [11], $R$ is a primitive ring which is isomorphic to a dense ring of linear transformations of a vector space $V$ over a division ring $D$. If $\operatorname{dim}_{D} V=\infty$, then, by Lemma 3 , we get the conclusion required.

Therefore consider the case $\operatorname{dim}_{D}(V)=k$, with $k$ finite positive integer $\geq 2$, because $R$ is not a domain. In this condition $R$ is a simple ring which satisfies a non-trivial generalized polynomial identity. By [7, Lemma 2; 12 theorem 2.3.29] $R \subseteq M_{t}(F)$, for a suitable field $F$ and $t \geq 2$, moreover $M_{t}(F)$ satisfies the same generalized identity of $R$. Since $f\left(x_{1}, . ., x_{n}\right)$ is not central on $R$ then, by [10], there exist $u_{1}, . ., u_{n} \in M_{t}(F)$, such that $f\left(u_{1}, . ., u_{n}\right)=\beta e_{i j}$, for some distinct $i, j$, with $\beta \in F-\{0\}$ and $e_{i j}$ the usual matrix unit with 1 in $(i, j)$-entry and zero elsewhere. Moreover, since the set $\left\{f\left(x_{1}, . ., x_{n}\right): x_{1}, . ., x_{n} \in M_{t}(F)\right\}$ is invariant under the action of all $F$-automorphisms of $M_{t}(F)$, then for any $i \neq j$ there exist $r_{1}, . ., r_{n} \in M_{t}(F)$ such that $f\left(r_{1}, . ., r_{n}\right)=\beta e_{i j}$.

Suppose on the contrary that the matrix $a=\sum a_{h l} e_{h l}$ is not zero. Let $q=$ $\sum q_{h l} e_{h l}$, with $q_{h l} \in F$ and fix $i$ and $j \neq i$. Then

$$
\begin{aligned}
0 & =a\left[q, f\left(r_{1}, . ., r_{n}\right)\right]^{m}=a\left(q f\left(r_{1}, . ., r_{n}\right)-f\left(r_{1}, . ., r_{n}\right) q\right)^{m} \\
& =a\left(q \beta e_{i j}-\beta e_{i j} q\right)^{m} .
\end{aligned}
$$

In particular, right multiplying by $e_{i j} q$ we have

$$
0=a\left(q \beta e_{i j}-\beta e_{i j} q\right)^{m} e_{i j} q=a(-\beta)^{m}\left(e_{i j} q\right)^{m+1} .
$$

Then, for all $j \neq i$, either $q_{j i}=0$ or the i-th column of the matrix $a$ is zero, a desired contradiction.

Case 1: $t=2$.

Since $f\left(x_{1}, . ., x_{n}\right)$ is not central on $R$, by [10, lemmas 2 and 9], there exists a sequence of matrices $r=\left(r_{1}, . ., r_{n}\right)$ such that $f(r)=\beta e_{21}$ is not zero.

Suppose that $q$ is not diagonal, say $q_{12} \neq 0$, then the 2-nd column of $a$ is zero. In other words the following hold:

$$
\begin{gathered}
q=\left[\begin{array}{ll}
q_{11} & q_{12} \\
q_{21} & q_{22}
\end{array}\right], \quad q_{12} \neq 0 \\
a=\left[\begin{array}{ll}
a_{11} & 0 \\
a_{21} & 0
\end{array}\right]
\end{gathered}
$$




$$
f(r)=\left[\begin{array}{ll}
0 & 0 \\
\beta & 0
\end{array}\right]
$$

By calculation it follows that

$$
[q, f(r)]^{2 m}=\left[\begin{array}{cc}
\left(q_{12} \beta\right)^{2 m} & 0 \\
0 & \left(q_{12} \beta\right)^{2 m}
\end{array}\right] .
$$

Then $0=a[q, f(r)]^{2 m}=a\left(q_{12} \beta\right)^{2 m}=0$ and so $a=0$.

Moreover we get the same conclusion if suppose $q_{21} \neq 0$. Thus we conclude that if $k=2$, either $q$ is a diagonal matrix or $a=0$.

Case 2: $t \geq 3$.

Also in this case we want to prove that if $a$ is not zero then $q$ is a diagonal matrix. Suppose there exists $q_{j i} \neq 0, i \neq j$, then the $i$-th column of $a$ is zero. For all $l \neq i, j$ let $\varphi_{l i} \in \operatorname{Aut}_{F}\left(M_{t}(F)\right)$ such that $\varphi_{l i}(x)=\left(1+e_{l i}\right) x\left(1-e_{l i}\right)$. Consider the following valutations of $f\left(x_{1}, . ., x_{n}\right)$ :

$$
f(r)=\gamma e_{i j}, \quad f(s)=\varphi_{l i}(f(r))=\gamma e_{i j}+\gamma e_{l j}, \quad \gamma \neq 0 .
$$

Since the $i$-th column of $a$ is zero, by $a[q, f(s)]^{m}=0$ and right multiplying by $e_{i j}+e_{l j}$, we have:

$$
0=a[q, f(s)]^{m}\left(e_{i j}+e_{l j}\right)=a(-\gamma)^{m}\left(q_{j i}+q_{j l}\right)^{m}\left(e_{i j}+e_{l j}\right)
$$

Notice that if $q_{j i}+q_{j l}=0$, then $q_{j l}=-q_{j i} \neq 0$ and, as in the first part of the proof, the $l$-th column of $a$ is zero. On the other hand, if $q_{j i}+q_{j l} \neq 0$, by (1), for all $k, a_{k l}=-a_{k i}$ and, since the $i$-th column of $a$ is zero, it follows again that the $l$-th one is also zero. Hence we can say that the matrix $a$ has at most one nonzero column, the $j$-th one.

Thus $a=a e_{j j}$ and so

$$
\begin{aligned}
0 & =a[q, f(r)]^{m}=a e_{j j}\left[q, \gamma e_{i j}\right]^{m}=a e_{j j}\left(q \gamma e_{i j}-\gamma e_{i j} q\right)\left[q, \gamma e_{i j}\right]^{m-1} \\
& =a e_{j j} q \gamma e_{i j}\left[q, \gamma e_{i j}\right]^{m-1}=\ldots . .=a e_{j j}\left(q \gamma e_{i j}\right)^{m}=a\left(q_{j i} \gamma\right)^{m} .
\end{aligned}
$$

Hence $a=0$.

The previous two cases show that if $a$ is the nonzero matrix then $q$ is a diagonal one, $q=\sum q_{k k} e_{k k}$. Now let $\varphi_{i j} \in A u t_{F}\left(M_{t}(F)\right)$ such that $\varphi_{i j}(x)=$ $\left(1+e_{i j}\right) x\left(1-e_{i j}\right)$, with $i \neq j$. Since $0=\varphi_{i j}(a)\left[\varphi_{i j}(q), \varphi\left(f\left(x_{1}, . ., x_{n}\right)\right)\right]^{m}=$ $\varphi_{i j}(a)\left[\varphi_{i j}(q), f\left(y_{1}, . ., y_{n}\right)\right]^{m}$ and $a \neq 0$, we have that $\varphi(q)$ is also diagonal. On the other hand $\varphi_{i j}(q)=q+\left(q_{j j}-q_{i i}\right) e_{i j}$, i.e. $q_{j j}=q_{i i}$ and $q$ is central in $M_{t}(F)$, which is a contradiction. Therefore must be $a=0$. 


\section{Acknowledgement}

The author wishes to thank the referee; by following his valuable suggestions, he has been able to shorten the proof of Lemmas 2 and 3 and to correct the proof of case 2 in Theorem 1.

\section{REFERENCES}

1. K. I Beidar, W. S. Martindale III, A. V. Mikhalev, Rings with generalized identities, Pure and Applied Math., Dekker, New York, 1996.

2. M. Bresar, A note on derivations, Math. J. Okayama Univ. 32 (1990), 83-88.

3. C. M. Chang and T. K. Lee, Annihilators of power values in prime rings, Comm. in Algebra, 26(7) (1998), 2091-2113.

4. C. L. Chuang, GPIs having coefficients in Utumi quotient rings, Proc. Amer. Mat. Soc. 103(3), (1988), 723-728.

5. C. L. Chuang and T. K. Lee, Rings with annihilators conditions on multilinear polynomials, Chin. J. Math. 24(2) (1996), 177-185.

6. V. K. Kharchenko, Differential identities of prime rings, Algebra and Logic 17 (1978), 155-168.

7. C. Lanski, An Engel condition with derivation, Proc. Amer. Math. Soc. 118(3) (1993), 731-734.

8. T. K. Lee, Semiprime ring with differential identities, Bull. Inst. Math. Acad. Sinica, 20(1) (1992), 27-38.

9. T. K. Lee and J. S. Lin, A result on derivations, Proc. Amer. Math. Soc. 124(6) (1996), 1687-1691.

10. U. Leron, Nil and power central polynomials in rings, Trans. Amer. Math. Soc. 202 (1975), 97-103.

11. W. S. Martindale III, Prime rings satisfying a generalized polynomial identity, $J$. Algebra 12 (1969), 576-584.

12. L. Rowen, Polynomial identities in Ring Theory, Pure and Applied Math. vol. 84 (1980), Academic Press, New York.

13. T. L. Wong, Derivations with power central values on multilinear polynomials, $\mathrm{Al}$ gebra Colloq. 3(4) (1996), 369-378.

Dipartimento di Matematica, Universitá di Messina

Salita Sperone, contrada Papardo

98166, Messina, ITALIA.

E-mail: enzo@dipmat.unime.it 